Research Paper

\title{
The Effects and Mechanism of YK-4-279 in Combination with Docetaxel on Prostate Cancer
}

\author{
Lin $\mathrm{Yu}^{1}$, Xiaofeng $\mathrm{Wu}^{1}$, Min Chen ${ }^{1}$, Huarong Huang ${ }^{1}$, Yan $\mathrm{He}^{1}$, Huaqian Wang ${ }^{1}$, Dongli Li², Zhiyun $\mathrm{Du}^{1}$, \\ Kun Zhang1,2, Susan Goodin ${ }^{3}$, Xi Zheng1,4凶 \\ 1. Allan H Conney Laboratory for Anticancer Research, School of Chemical Engineering and Light Industry, Guangdong University of Technology, \\ Guangzhou 510006, China; \\ 2. School of Chemical and Environmental Engineering, Wuyi University, Jiangmen 529020, China; \\ 3. Rutgers Cancer Institute of New Jersey, New Brunswick, NJ 08903, USA; \\ 4. Department of Chemical Biology, Ernest Mario School of Pharmacy, Rutgers, The State University of New Jersey, Piscataway, NJ 08854, USA.
}

$\triangle$ Corresponding author: Xi Zheng, Allan H Conney Laboratory for Anticancer Research, School of Chemical Engineering and Light Industry, Guangdong University of Technology, Guangzhou 510006, China; Department of Chemical Biology, Ernest Mario School of Pharmacy, Rutgers, The State University of New Jersey, Piscataway, NJ 08854, USA. Phone: 848-445-8069 Email: xizheng@pharmacy.rutgers.edu

(c) Ivyspring International Publisher. This is an open access article distributed under the terms of the Creative Commons Attribution (CC BY-NC) license (https://creativecommons.org/licenses/by-nc/4.0/). See http://ivyspring.com/terms for full terms and conditions.

Received: 2016.11.16; Accepted: 2017.03.14; Published: 2017.04.07

\begin{abstract}
Background: Docetaxel is the first-line treatment for castration-resistant prostate cancer (CRPC). The limited survival benefit associated with the quick emergence of resistance and systemic toxicity diminishes its efficacy in high-dose monotherapy. YK-4-279 is a small molecule inhibitor of ETVI that plays an important role in the progression of prostate cancer. The aim of this study was to evaluate the hypothesis that the combination of docetaxel and YK-4-279 will have a synergistic effect on inhibiting growth and accelerating apoptosis in human prostate cancer cells.

Methods: Cell growth assessed using CCK-8 and trypan blue exclusion assays. Cell apoptosis was determined by morphological assessment in cells stained with propidium iodide. Standard scratch migration and Matrigel-coated transwell invasion assays were used to assess cell migration and invasion, respectively. Western blotting was used to investigate the levels of ETVI, AR, PSA, P-STAT3, survivin, Bcl-2, and p-Akt in prostate cancer cells.

Results: The combination of low-dose docetaxel and YK-4-279 synergistically inhibited growth and induced apoptosis in human prostate cancer cells. The combination also more efficiently suppressed the migration and invasion of $\mathrm{LNCaP}$ and PC-3 cells. The combination of low-dose docetaxel and YK-4-279 caused a stronger decrease in the levels of ETV1, AR, PSA, P-STAT3, survivin, Bcl-2, and p-Akt in LNCaP cells and of p-Akt, Bcl-2, and p-STAT3 in PC-3 cells compared with either drug alone.

Conclusions: These data suggest that the combination of docetaxel and YK-4-279 may be an effective approach for inhibiting the growth and metastasis of prostate cancer. This could permit a decrease in the docetaxel dose necessary for patients with CRPC and thereby lower its systemic toxicity.
\end{abstract}

Key words: docetaxel, YK-4-279, prostate cancer, combination, synergistic action.

\section{Introduction}

Prostate cancer is the most frequently occurring cancer and the second leading cause of cancer-related deaths among men in the United States [1]. Patients diagnosed with localized stage disease are sensitive to various treatments and are often curable; however, $\sim 40 \%$ of all cases will eventually progress to a metastatic stage [2].

Since the 1940s, targeting androgen signaling using androgen deprivation therapy (ADT) has been the mainstay of clinical interventions for metastatic hormone-sensitive prostate cancer [3]. However, its initial effectiveness is only transient (2-3 years) [4], 
and most men relapse with castration-resistant prostate cancer (CRPC) and die soon thereafter [5]. For patients with CRPC, the common treatment is docetaxel-based chemotherapy to prolong survival and maintain a good quality of life [6]. However, treatment using high doses of docetaxel ultimately cause toxicity and resistance, which means that there are only limited options for patients progressing on or after docetaxel [7]. Recently, some novel agents including abiraterone, cabazitaxel, and enzalutamide were approved for patients with CRPC following docetaxel failure or resistance, but their efficiency is limited [8]. Therefore, there is an urgent need to improve docetaxel-based regimens to reduce toxicity and increase efficacy.

Prostate cancer that progresses to lethal CRPC has been associated with ETS gene fusions, PTEN loss, and androgen receptor (AR) amplification [9]. In particularly, ETS transcription factor (mainly ETV1 or ERG) fusions occur frequently in prostate cancer [10-11], and $\sim 50 \%$ of human prostate cancers containing ETS gene fusions [12]. One study suggested that ETV1 expression promotes autonomous testosterone production to reactivate $A R$ signaling in aggressive disease [13]. It was suggested that ETV1 plays an important role in the progression of CRPC and can indirectly mediate AR signaling.

YK-4-279 monotherapy can inhibit the growth and metastasis of ETV1 fusion-positive prostate cancer xenografts [14-15]. This suggests that YK-4-279 could be used as a small molecule inhibitor of ETV1. Docetaxel, a semi-synthetic second-generation taxane, can slow down the progression of prostate cancer, and it retains antitumor activity in CRPC patients [16]. It can inhibit proliferation and induce apoptosis by binding to $\beta$-tubulin and causing cell-cycle arrest [17].

The addition of docetaxel to ADT has survival benefits compared with ADT alone in patients with metastatic hormone-sensitive prostate cancer [18-19]. This finding suggests that there is an interaction between AR signaling and docetaxel sensitivity. Thus, we hypothesized that the combination of YK-4-279 and docetaxel will synergistically inhibit growth and accelerate apoptosis in human prostate cancer cells.

\section{Materials and methods}

\section{Cell culture and reagents}

Two human prostate cancer cell lines (LNCaP and PC-3) were obtained from the American Type Culture Collection (Rockville, MD, USA). YK-4-279 was purchased from MCE (MedChem Express, HY-1450, USA) and docetaxel was from Aladdin (D107319). The cells were cultured as described previously [20]. Docetaxel and YK-4-279 were dissolved in DMSO (Sigma, USA); the final concentration of DMSO was $0.1 \%$ in all experiments.

\section{Cell viability}

For CCK-8 assays [21], cells were seeded at a density of $2 \times 10^{4}$ cells $/ \mathrm{ml}$ of medium in a 96-well plate $(0.1 \mathrm{ml} /$ well $)$ and incubated for $24 \mathrm{~h}$. Then, the cells were treated with different concentrations of docetaxel $(1 \mathrm{nM})$ and YK-4-279 $(0.1 \mu \mathrm{M}, 0.5 \mu \mathrm{M}$, or 1.0 $\mu \mathrm{M})$ for $72 \mathrm{~h}$. After treatment, the media were replaced with fresh media and $10 \mu \mathrm{l} \mathrm{CCK-8} \mathrm{(Dojindo,}$ Kumamoto, Japan) was added to each well. After a 1-h incubation, the absorbance at $450 \mathrm{~nm}$ was measured on a microplate reader.

For the trypan blue exclusion assays [22], cells were seeded at a density of $2 \times 10^{4}$ cells $/ \mathrm{ml}$ of medium in $35-\mathrm{mm}$ tissue culture dishes $(2 \mathrm{ml} /$ dish $)$ and incubated for $24 \mathrm{~h}$. The cells were then treated with different concentrations of docetaxel $(0.1 \mathrm{nM}, 0.5 \mathrm{nM}$, or $1 \mathrm{nM})$ and YK-4-279 $(0.1 \mu \mathrm{M}, 0.5 \mu \mathrm{M}$, or $1.0 \mu \mathrm{M})$ for $72 \mathrm{~h}$. Then, single cell suspensions were prepared and the number of viable cells was counted using a hemocytometer under a light microscope by mixing $80 \mu \mathrm{l}$ of cell suspension and $20 \mu \mathrm{l}$ of $0.4 \%$ trypan blue solution for $5 \mathrm{~min}$. Blue cells were counted as dead and the cells that did not absorb dye were counted as live.

\section{Measuring apoptosis}

Apoptosis was determined by morphologically assessing cells stained with propidium iodide (PI) [22]. Cells were seeded at a density of $2 \times 10^{4}$ cells $/ \mathrm{ml}$ in $35-\mathrm{mm}$ tissue culture dishes $(2 \mathrm{ml} /$ dish $)$ and incubated for $24 \mathrm{~h}$. They were then treated with docetaxel $(0.5 \mathrm{nM})$ and/or YK-4-279 $(0.5 \mu \mathrm{M})$ for $72 \mathrm{~h}$. After treatment, cytospin slides were prepared using a smear centrifuge and fixed with acetone/methanol (1:1) for $10 \mathrm{~min}$ at room temperature. The cells were stained with $1 \mu \mathrm{g} / \mathrm{ml}$ PI in PBS for $10 \mathrm{~min}$. Then we identified apoptotic cells using a fluorescence microscope. Cells with classical morphological features including nuclear condensation, cell shrinkage, and the formation of apoptotic bodies were counted as apoptotic. At least 400 cells in approximately 10 randomly selected fields were counted in each sample.

\section{Scratch migration assays}

For scratch migration assays [23], cells were seeded at a density of $5 \times 10^{5}$ cells $/ \mathrm{ml}$ in $35-\mathrm{mm}$ tissue culture dishes ( $2 \mathrm{ml} /$ dish) and incubated for $24 \mathrm{~h}$. The cell surface was then scratched using a sterile $200-\mu 1$ pipette tip (Axygen, Union City, CA, USA) after washing with PBS. Then, the scratched cells were rinsed gently with PBS three times and complete medium was added $(0.2 \%$ FBS). The cells were then 
treated with different concentrations of docetaxel and/or YK-4-279 for an additional $24 \mathrm{~h}$. Images were captured using an inverted microscope. The distance that cells migrated compared with baseline measurements was measured using Image J software.

\section{Invasion assays}

For Matrigel-coated transwell invasion assays [24], $600 \mu$ l complete medium (20\% FBS) was added to the lower chamber. The cells were pre-treated with YK-4-279 or docetaxel for $48 \mathrm{~h}$. Then, the cells were trypsinized, resuspended in complete medium $(1 \%$ FBS), and seeded at a density of $5 \times 10^{5}$ cells $/ \mathrm{ml}$ in the top chamber (200 $\mu \mathrm{l} /$ chamber; Corning) containing a Matrigel-coated membrane. The cells were then treated with different concentrations of docetaxel and/or YK-4-279 for an additional $24 \mathrm{~h}$. Next, the medium and the cells remaining in the top chambers were removed. After fixing with methanol and staining with $0.1 \%$ crystal violet, the number of cells that had invaded to the lower membrane was counted and images were captured under an inverted microscope (Olympus).

\section{Western blotting}

After treatment, the protein lysates were prepared as described previously [25]. Proteins were separated by sodium dodecyl sulfate polyacrylamide gel electrophoresis (SDS-PAGE) and transferred to polyvinylidene fluoride (PVDF) membranes (Millipore). After blocking nonspecific binding sites with blocking buffer, the membranes were incubated overnight at $4^{\circ} \mathrm{C}$ with the following primary antibodies: \#4060 for phospho-Akt, \#9145 for phospho-Stat3, \#5365 for PSA/KLK3, \#2808 for survivin, \#2870 for Bcl-2, \#4370 for phospho-p44/42 MAPK (ERK-1/2), \#12153 for IL-6, \#3202 for AR (all from Cell Signaling Technology, Beverly, MA), and ab81086 for ETV1 (Abcam, Cambridge, MA, USA). $\beta$-actin (Cell Signaling Technology, Beverly, MA) was used as a loading control. Following removal of the primary antibody, the membranes were washed three times with TBST (TBS containing 0.05\% Tween 20) at room temperature and then incubated with fluorochrome-conjugated secondary antibody for $2 \mathrm{~h}$. The membranes were then washed with TBST three times and signals were detected using ECL in the dark room.

\section{Statistical analysis}

The potential synergistic effects of docetaxel and YK-4-279 were assessed using the isobole method with the equation $\mathrm{Ac} / \mathrm{Ae}+\mathrm{Bc} / \mathrm{Be}=$ combination index (CI) [26]. Ac and Bc represent the concentration of drug $\mathrm{A}$ and drug $\mathrm{B}$ used in the combination, respectively, and Ae and Be represent the concentration of drug A and B that produced the same magnitude of effect when administered alone. If $\mathrm{CI}$ is $<1$, then the drugs are considered to act synergistically; if $\mathrm{CI}$ is $>1$ or $=1$, then the drugs act in an antagonistic or additive manner, respectively. Comparisons of cell viability, apoptosis, migration, and invasion were analyzed using ANOVA with Tukey-Kramer multiple comparison tests.

\section{Results}

\section{Effects of docetaxel and YK-4-279 alone or in combination on prostate cancer cell growth and apoptosis}

The effects of docetaxel and YK-4-279 alone or in combination on the growth of human prostate cancer cells were determined using the CCK-8 and trypan blue exclusion assays. Human LNCaP (androgen-dependent), PC-3 (androgen-independent) prostate cancer cells were treated with different concentrations of docetaxel and YK-4-279 for $72 \mathrm{~h}$. As shown in Fig. 1A and B, treatment with YK-4-279 (0.1 $\mu \mathrm{M}, 0.5 \mu \mathrm{M}$, and $1.0 \mu \mathrm{M})$ alone and in combination with docetaxel $(1 \mathrm{nM})$ inhibited the growth of both LNCaP and PC-3 cells in a dose-dependent manner. Treatment with the combination of docetaxel and YK-4-279 had a stronger inhibitory effect on cell growth than either drug alone. When the concentration ratio of YK-4-279 and docetaxel was 1:1000, co-treatment with YK-4-279 and docetaxel exhibited a stronger decrease in cell viability compared with the other two combination groups. As shown in Fig. 1C and D, docetaxel and YK-4-279 single and combined treatments affected the viability of LNCaP and PC-3 cells in a dose-dependent manner. The half maximal inhibitory concentrations (IC50) of YK-4-279 were $1.48 \mu \mathrm{M}$ and $2.03 \mu \mathrm{M}$ in LNCaP and PC-3 cells, respectively, and the IC50 of docetaxel alone was $1.16 \mathrm{nM}$ and $2.07 \mathrm{nM}$ in LNCaP and PC-3 cells, respectively. Specifically, the IC50 of docetaxel was decreased to $0.41 \mathrm{nM}$ and $0.66 \mathrm{nM}$ in LNCaP and PC-3 cells respectively, whereas those of YK-4-279 decreased to $0.41 \mu \mathrm{M}$ and $0.66 \mu \mathrm{M}$. The combination indexes (CIs) for the IC50 were 0.64 and 0.64 in LNCaP and PC-3 cells, respectively. These results suggest that the combination of YK-4-279 and docetaxel synergistically inhibited the growth of both LNCaP and PC-3 cells.

Next, the effects of docetaxel and YK-4-279 alone or in combination on prostate cancer cell apoptosis were determined by morphologically assessing cells stained with PI. Apoptotic cells were identified according to classic morphologic features such as nuclear condensation, cell shrinkage, and the formation of apoptotic bodies [23]. Morphologically 
distinct apoptotic cells from representative samples are shown in Fig. 2. As shown in Table 1, treating cells with docetaxel $(0.5 \mathrm{nM})$ and YK-4-279 $(0.5 \mu \mathrm{M})$ alone resulted in a small number of apoptotic cells. In contrast, the combination of docetaxel and YK-4-279 caused a strong increase in the number of apoptotic cells, suggesting that the combination of docetaxel and YK-4-279 had a more potent effect on stimulating apoptosis than either agent alone.

A

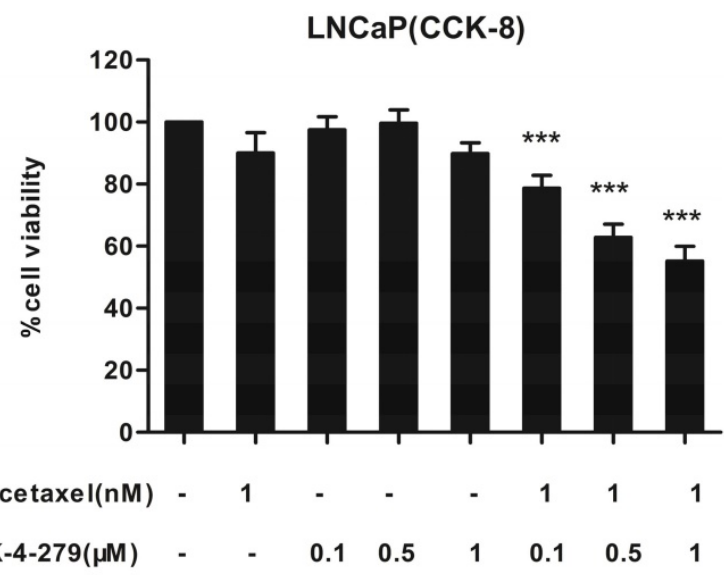

C

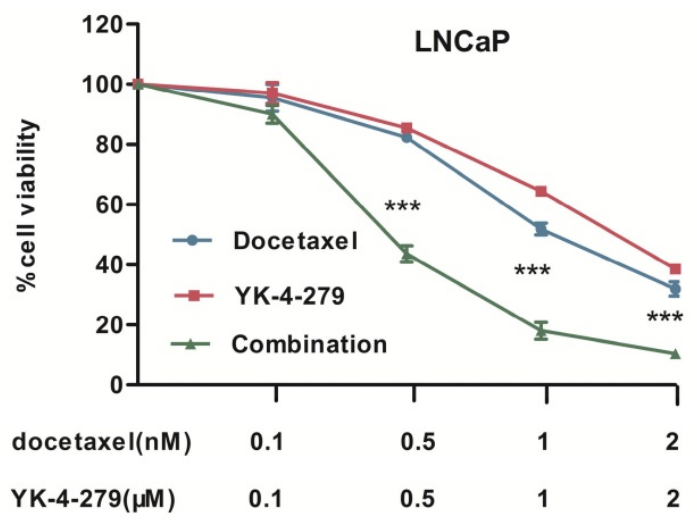

Table 1. Effect of docetaxel and YK-4-279 alone or in combination on LNCaP and PC-3 cell apoptosis

\begin{tabular}{lllll}
\hline \multirow{2}{*}{ Treatment } & \multicolumn{2}{l}{ Apoptotic cells (\%) } & \multicolumn{2}{l}{ Dead cells (\%) } \\
\cline { 2 - 5 } & LNCaP & PC-3 & LNCaP & PC-3 \\
\hline Control & $2.32 \pm 0.31$ & $3.38 \pm 0.89$ & $3.67 \pm 1.15$ & $4.01 \pm 0.55$ \\
Docetaxel & $4.95 \pm 0.57$ & $4.38 \pm 0.86$ & $5.33 \pm 0.58$ & $4.88 \pm 0.78$ \\
YK-4-279 & $5.52 \pm 0.84$ & $4.72 \pm 0.81$ & $6.67 \pm 0.58$ & $4.94 \pm 0.38$ \\
Combination & $17.03 \pm 3.12^{* * *}$ & $17.04 \pm 0.64^{* * *}$ & $18.67 \pm 0.15^{* * *}$ & $19.96 \pm 2.11^{* * *}$
\end{tabular}

LNCaP or PC-3 cells was cultured at a density of $2 \times 10^{4}$ cells $/ \mathrm{ml}$ for $24 \mathrm{~h}$. The cells were then treated with docetaxel $(0.5 \mathrm{nM})$ and $\mathrm{YK}-4-279(0.5 \mu \mathrm{M})$ alone or in combination for $72 \mathrm{~h}$. Apoptosis was determined by morphological assessment. Each value represents mean \pm S.E from three separate experiments. Significant numbers of apoptotic cells between a combination group and a single-agent-treated group were analyzed by ANOVA with Tukey-Kramer multiple comparison test $\left({ }^{*} \mathrm{p}<0.05,{ }^{* *} \mathrm{p}<0.01,{ }^{* * *} \mathrm{p}<0.001\right)$.

B

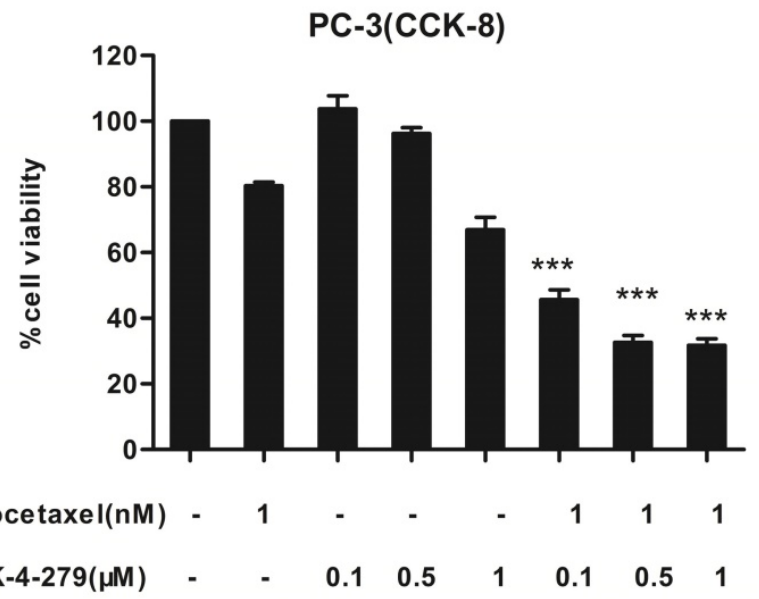

D

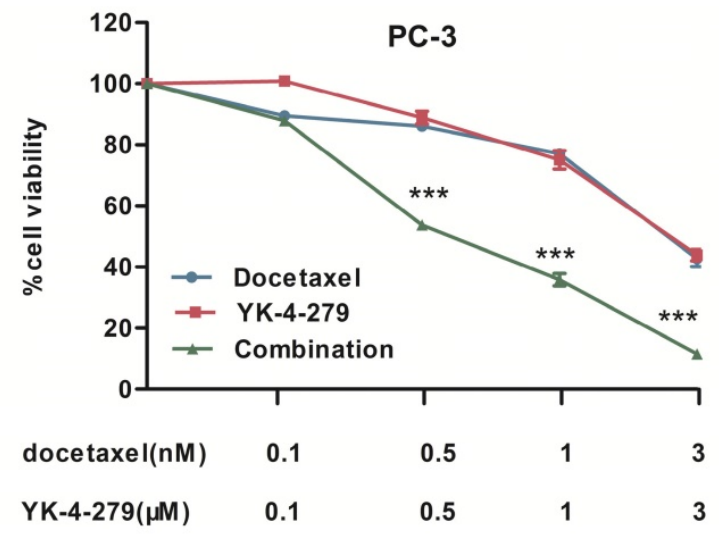

Figure 1. Effects of docetaxel and YK-4-279 alone or in combination on LNCaP and PC-3 cell growth. A and B. LNCaP or PC-3 cells was were seeded at a density of $2 \times 10^{4}$ cells $/ \mathrm{ml}$ of medium in a 96 -well plate $(0.1 \mathrm{ml} /$ well) and incubated for $24 \mathrm{~h}$. The cells were then treated with docetaxel or YK-4-279 for 72 hours and cell growth was evaluated by CCK-8 assay. C and D. LNCaP or PC-3 cells was cultured at a density of $2 \times 104$ cells/ml in $35 \mathrm{~mm}$ tissue culture dishes ( $2 \mathrm{ml} / \mathrm{dish})$ for $24 \mathrm{~h}$. The cells were then treated with docetaxel or YK-4-279 for $72 \mathrm{~h}$. The number of viable cells was determined by the tyrpan blue exclusion assay and expressed as percentages of solvent-treated control. Each value represents mean \pm S.E. from three separate experiments. Significant numbers of viable cells between a combination group and a single-agent-treated group were analyzed by ANOVA with Tukey-Kramer multiple comparison test $(* \mathrm{p}<0.05$, $* * \mathrm{p}<0.01, * * * \mathrm{p}<0.001)$. 
A

LNCaP

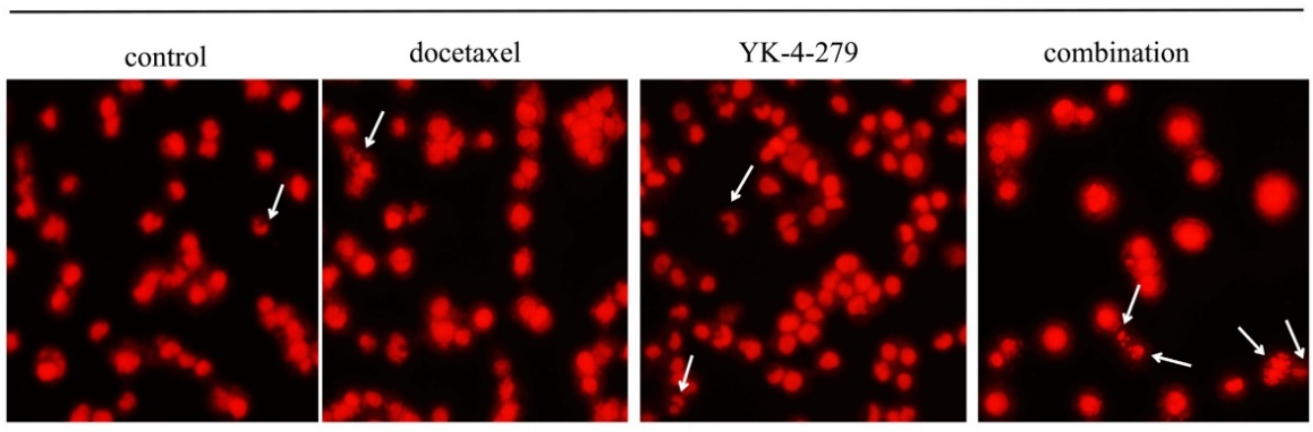

B

\section{PC-3}

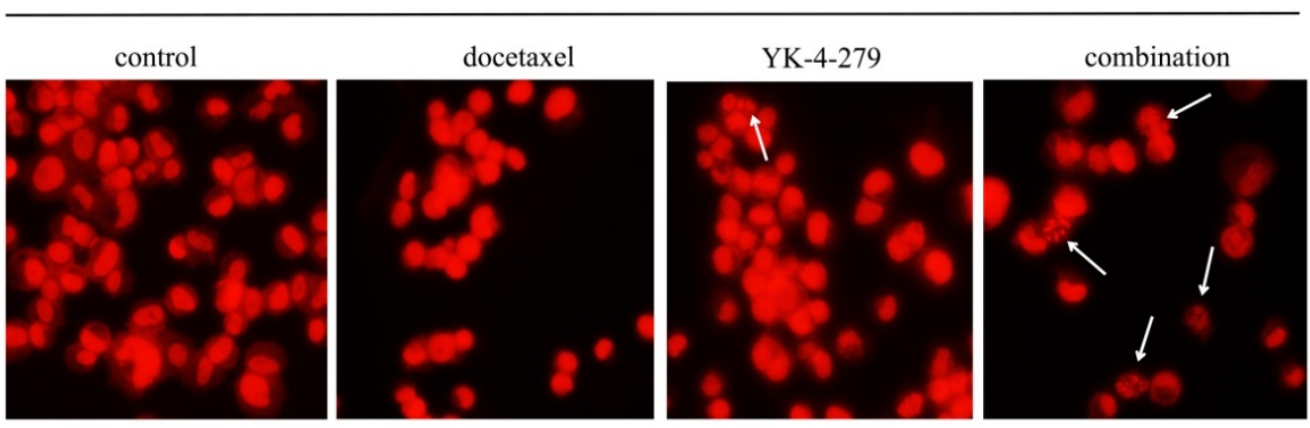

Figure 2. Effect of docetaxel and YK-4-279 alone or in combination on LNCaP and PC-3 cell apoptosis. The nuclear morphology changes were analyzed by fluorescence microscopy in $\times 200$ magnification using the propidium iodide nuclear fluorescent dye staining. These analyses were performed $72 \mathrm{~h}$ after treatment. Arrows represent the apoptotic cells. A: LNCaP cells, B: PC-3 cells.

\section{Effects of docetaxel and YK-4-279 alone or in combination on LNCaP and PC-3 cell motility}

The effects of docetaxel and YK-4-279 alone or in combination on the migration of LNCaP and PC-3 cells was determined by a standard scratch migration assay. Prior to performing this experiment, the effects of time on the growth of LNCaP and PC-3 cells treated with docetaxel and YK-4-279 alone or in combination was determined using trypan blue exclusion assays (not shown). The number of viable cells in the combination treatment group was comparable to the individual treatments within $24 \mathrm{~h}$; therefore, the co-treatment had no effects on the proliferation of LNCaP and PC-3 cells within 24 hours. These findings suggest that if a scratch assay was performed within $24 \mathrm{~h}$, the effects of the co-treatment would not be due to cytotoxicity but instead due to the inhibition of cell migration. Treating LNCaP and PC-3 cells with docetaxel and/or YK-4-279 had significantly different effects on the number of migrated cells (Fig. 3A and B). More cells in the control and single agent-treated groups migrated cells than in the combination treatment group. The combination of docetaxel $(0.5$ $\mathrm{nM})$ and YK-4-279 $(0.5 \mu \mathrm{M})$ caused an $88 \%$ and $77 \%$ decrease in the number of migrating $\mathrm{LNCaP}$ and PC-3 cells, respectively, compared with control (Fig. $3 \mathrm{C}$ and D). The combination of docetaxel $(0.5 \mathrm{nM})$ and YK-4-279 $(0.5 \mu \mathrm{M})$ had a more potent effect on inhibiting the migration of LNCaP cells compared with PC-3 cells.

\section{Effects of docetaxel and YK-4-279 alone or in combination on LNCaP and PC-3 cell invasion}

The effects of docetaxel and YK-4-279 alone or in combination on the invasiveness of LNCaP and PC-3 cells was determined using Matrigel-coated transwell invasion assays. There was a significant difference in the invasiveness of $\mathrm{LNCaP}$ and PC-3 cells treated with a single agent and the combination treatment (Fig. 4A and B). Treatment with docetaxel $(0.5 \mathrm{nM})$ and YK-4-279 $(0.5 \mu \mathrm{M})$ alone had little or no effect on the number of cells invading through the Matrigel-coated inserts. However, combination treatment led to a $40 \%$ and $36 \%$ decrease in the number of invading LNCaP and PC-3 cells, respectively, compared with control (Fig. 4C and D). The combination of docetaxel (0.5 $\mathrm{nM})$ and YK-4-279 $(0.5 \mu \mathrm{M})$ had more potent effect on inhibiting the invasion of LNCaP cells compared with PC-3 cells. 
A

LNCaP

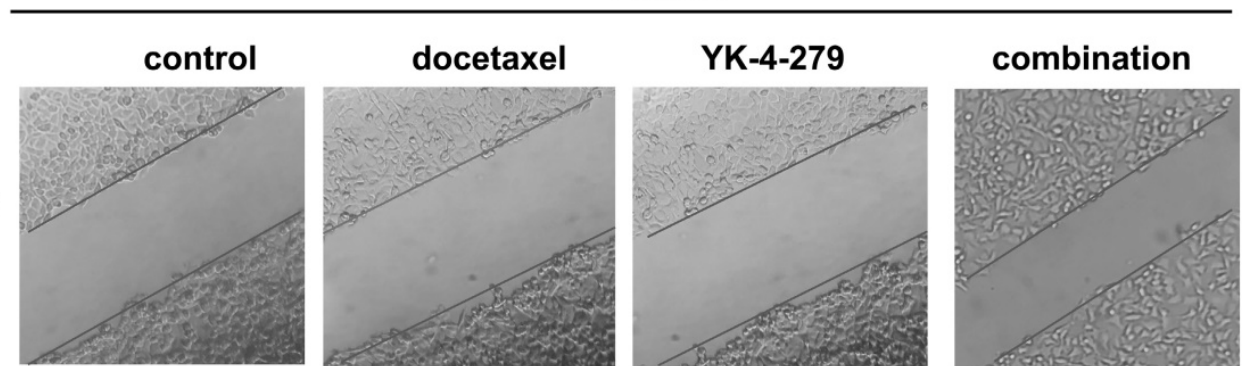

24h
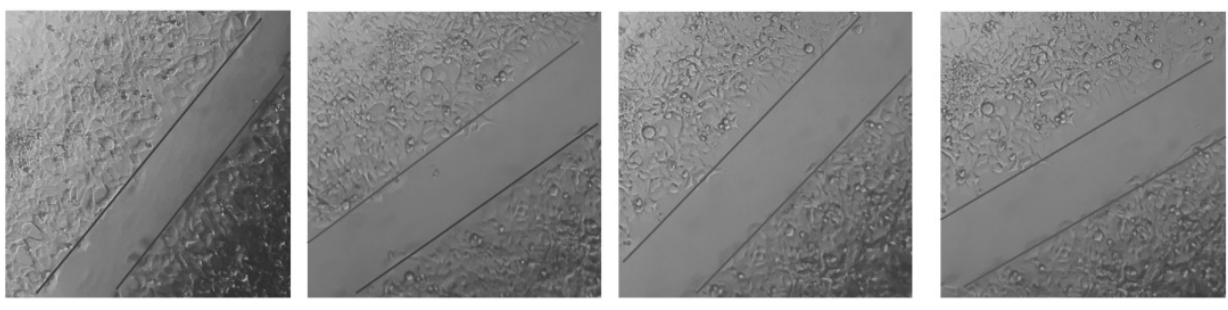

B

PC-3

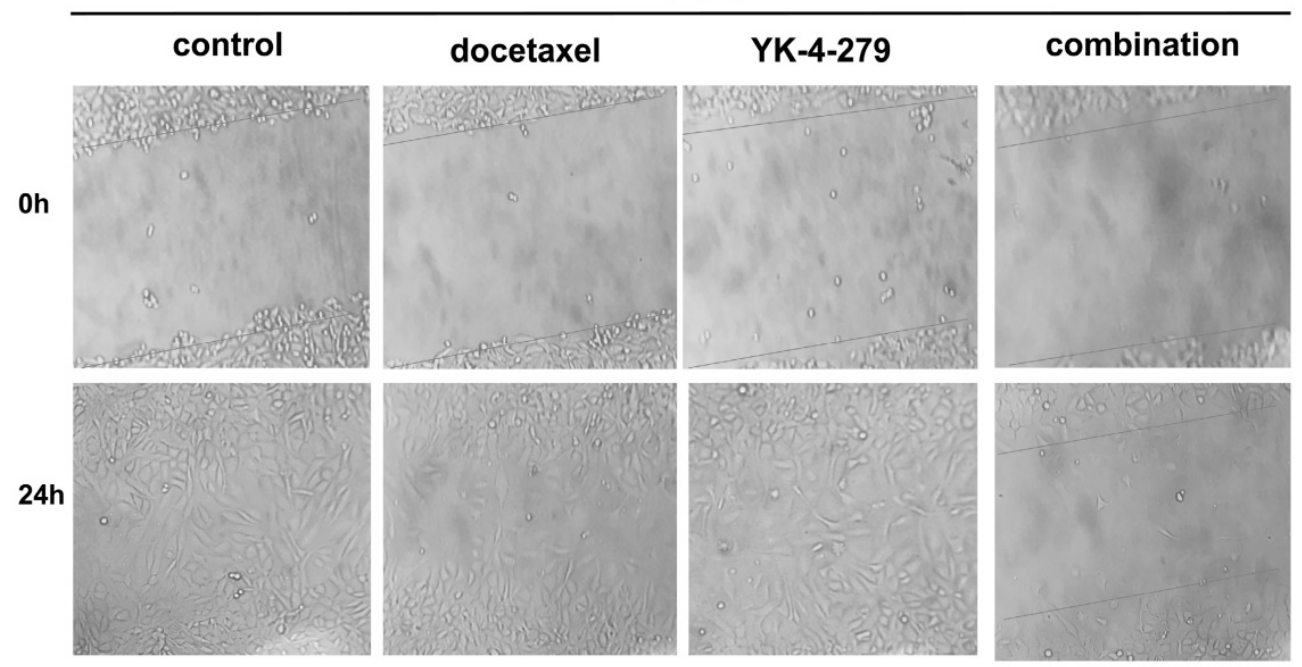

C

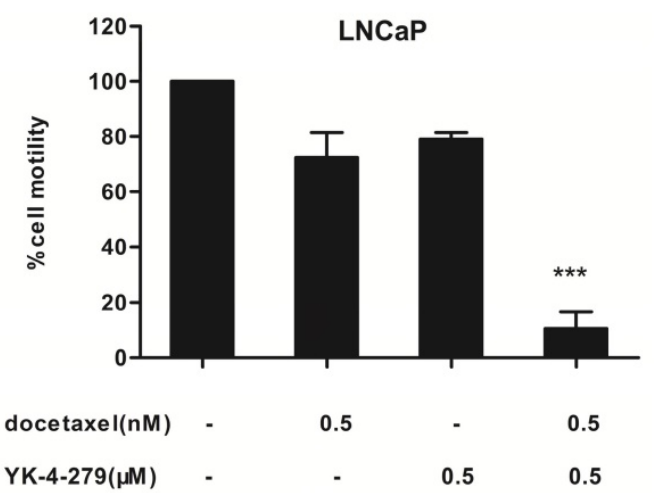

D

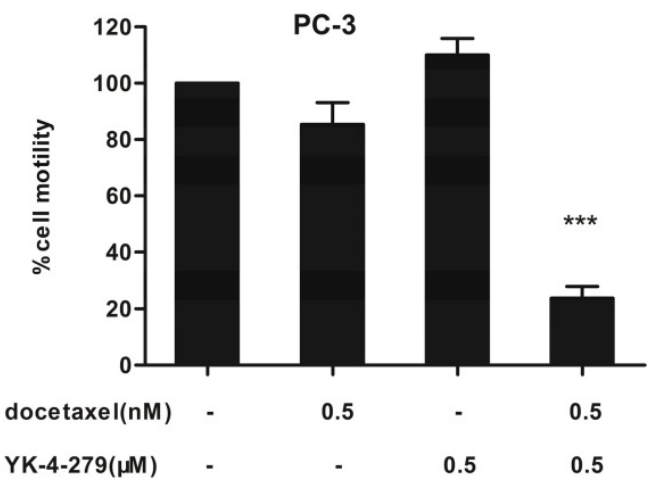

Figure 3. Effects of docetaxel and YK-4-279 alone or in combination on LNCaP and PC-3 cell motility. LNCaP and PC-3 cells were seeded at a density of $5 \times 10^{5}$ cells $/ \mathrm{ml}$ in $35 \mathrm{~mm}$ tissue culture dishes $(2 \mathrm{ml} / \mathrm{dish})$ for $24 \mathrm{~h}$. After the scratch finished, the cells were then treated with different concentrations of docetaxel and YK-4-279 alone or in combination for an additional 24h. Cell motility was quantified by measuring the distance between the migrating cell boundaries. Motility was expressed relative to vehicle treated conditions. Significant cell motility between a combination group and a single-agent-treated group were analyzed by ANOVA with Tukey-Kramer multiple comparison test $\left({ }^{*} \mathrm{p}<0.05, * * \mathrm{p}<0.01, * * * \mathrm{p}<0.001\right)$. 
A

\section{LNCaP}

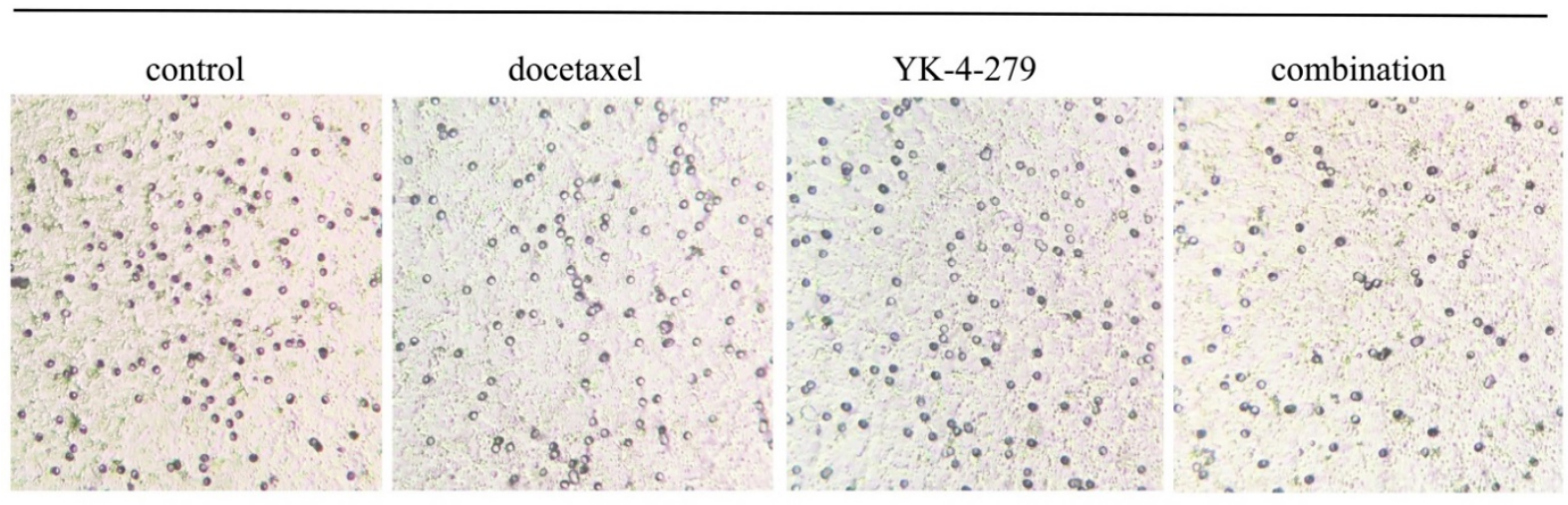

B

PC-3

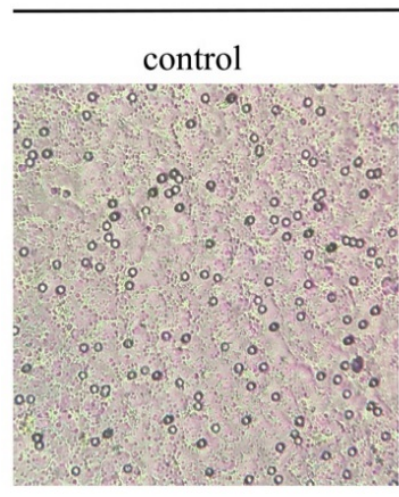

C

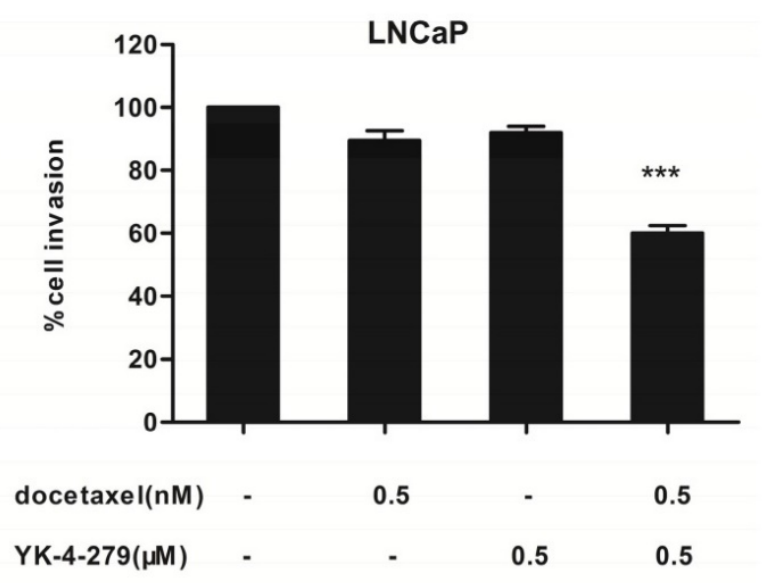

YK-4-279

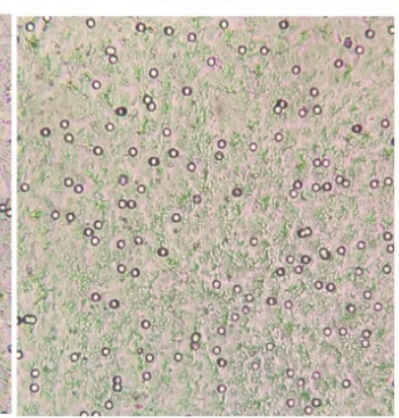

combination

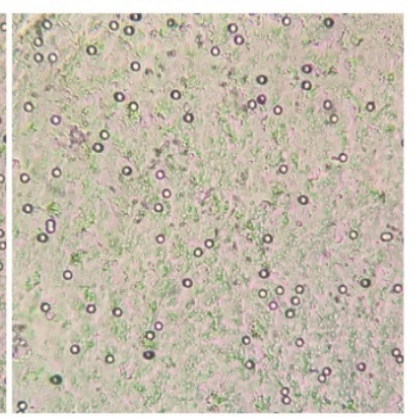

D

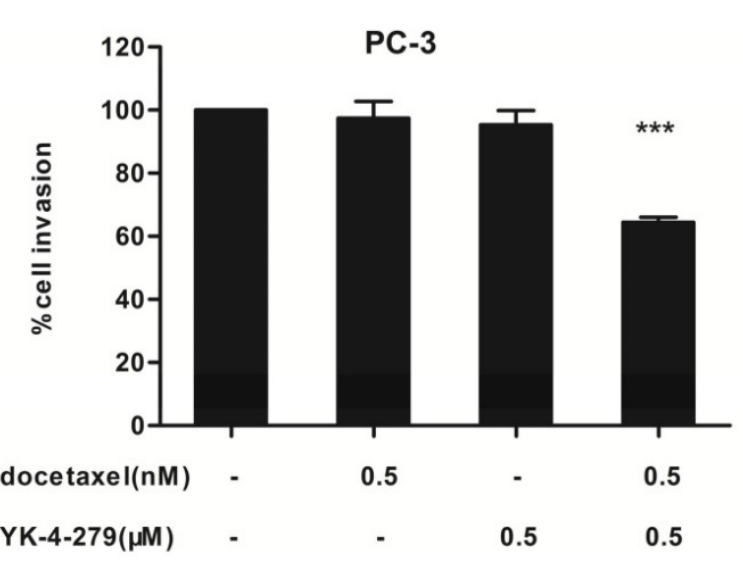

Figure 4. Effects of docetaxel and YK-4-279 alone or in combination on LNCaP and PC-3 cell invasion. LNCaP and PC-3 cells were pre-treated for 48 hours with YK-4-279 and docetaxel alone or in combination. The cells were trypsinized and resuspended in complete medium (1\%FBS) and seeded at a density of 5

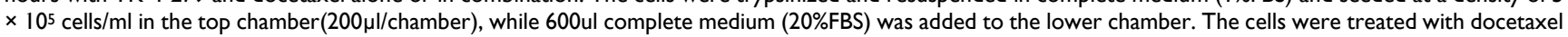
and YK-4-279 alone or in combination for an additional 24h.After fixation and staining, the cells that had invaded to the lower membrane of the inserts were counted and images were captured. Invasion was expressed relative to vehicle treated conditions. Significant invasion (\% of control) between a combination group and a single-agent-treated group were analyzed by ANOVA with Tukey-Kramer multiple comparison test $\left({ }^{*} \mathrm{p}<0.05,{ }^{* *} \mathrm{p}<0.01, * * * \mathrm{p}<0.001\right)$. 


\section{Effects of docetaxel and YK-4-279 alone or in combination on the levels of ETV1, AR, PSA, p-STAT3, survivin, Bcl-2 and p-Akt in LNCaP and PC-3 cells}

The levels of ETV1, AR, PSA, p-STAT3, survivin, $\mathrm{Bcl}-2$, and p-Akt in LNCaP and PC-3 cells were determined using western blotting. The PI3K/Akt/m TOR signaling pathway has many functions, including the regulation of cellular growth, proliferation, migration, and angiogenesis [27]. It also plays an important role in facilitating prostate cancer progression to CRPC and is highly activated in prostate cancer [28]. Low-dose docetaxel alone did not alter the expression of p-Akt in either LNCaP or PC-3 cells whereas the combination treatment strongly decreased expression in both cell lines, suggesting that YK-4-279 functions as a docetaxel chemosensitizer. As shown in Fig. 5, in LNCaP cells, the level of p-Akt relative to control (1.00) was 0.91 in cells treated with docetaxel, 0.75 in cells treated with YK-4-279, and 0.44 in cells treated with the combination of docetaxel and YK-4-279. In PC-3 cells, the level of p-Akt was 1.00 in control, 0.94 in cells treated with docetaxel, 0.51 in cells treated with

YK-4-279, and 0.36 in cells treated with the combination.

Bcl-2 family members are important in the regulation and control of the intrinsic apoptosis pathway. Although the exact mechanism of action of docetaxel is not well-understood, it is believed to inhibit Bcl-2 and Bcl-x activity by decreasing their gene expression to promote apoptosis in prostate cancer cells [29]. In the current study, the expression of Bcl-2 was significantly decreased in cells treated with the combination of docetaxel and YK-4-279 compared with the control in both LNCaP and PC-3 cells. This suggests that decreasing Bcl-2 levels may play a role in the apoptosis induced by the combination of docetaxel and YK-4-279. As shown in Fig. 5, in LNCaP cells the levels of Bcl-2 relative to control (1.00) was 0.94 in cells treated with docetaxel, 0.80 in cells treated with YK-4-279, and 0.42 in cells treated with the combination of docetaxel and YK-4-279. In PC-3 cells, the level of Bcl-2 relative to control (1.00) was 0.93 in cells treated with docetaxel, 0.79 in cells treated with YK-4-279, and 0.31 in cells treated with the combination.

\section{LNCaP}

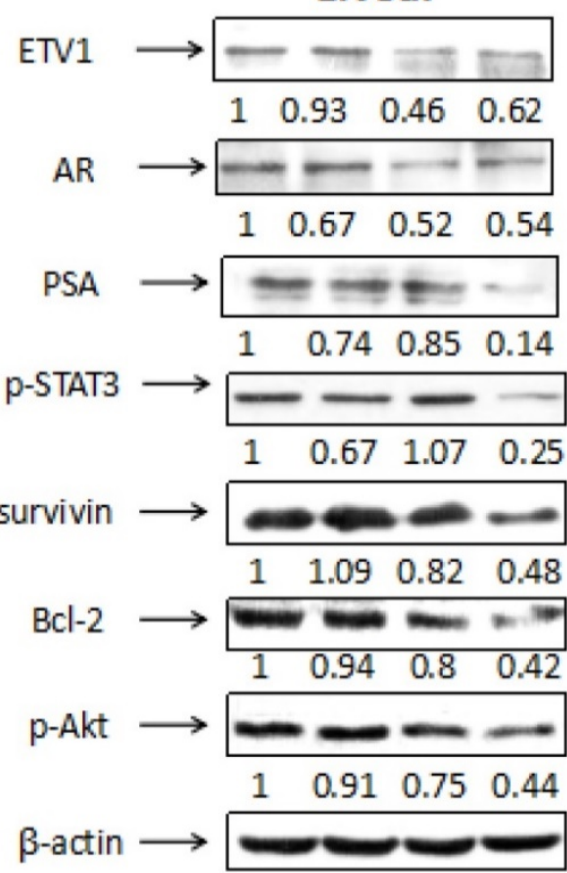

$\begin{array}{lllll}\text { docetaxel }(0.5 \mathrm{nM}) & - & + & - & + \\ \mathrm{YK}-4-279(0.5 \mu \mathrm{M}) & - & - & + & +\end{array}$

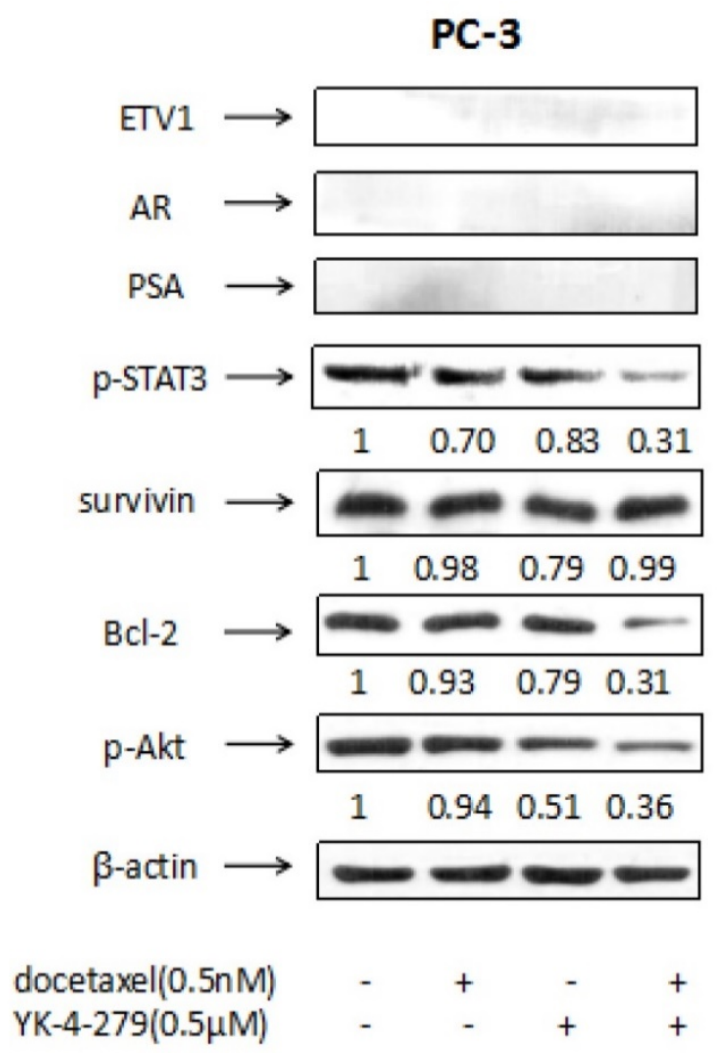

Figure 5. Effects of docetaxel and YK-4-279 alone or in combination on the levels of ETV1, AR, PSA, p-STAT3, survivin, Bcl-2 and p-Akt in LNCaP and PC-3 cells. LNCaP and PC-3 cells were cultured at a density of $1 \times 105$ cells $/ \mathrm{ml}$ in $100 \mathrm{~mm}$ tissue culture dishes $(10 \mathrm{ml} / \mathrm{dish})$ for $24 \mathrm{~h}$. The cells were

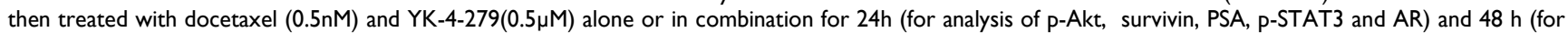
analysis of Bcl-2). The levels of AR,Bcl-2, p-STAT3, PSA,p-Akt and survivin were determined by the Western blot analysis. The band density was measured and normalized for actin. 
Survivin is a member of the inhibitor of apoptosis protein family and has been actively pursued as a target for cancer treatment because its overexpression is correlated to recurrence, metastasis, and therapeutic resistance [30]. Moreover, accumulating evidence suggests that survivin plays a pivotal role in the progression of prostate cancer [31]. In the current study, the combination of docetaxel and YK-4-279 significantly suppressed survivin expression in LNCaP cells but not in PC-3 cells, suggesting that the mechanism of action of the combination treatment differs in LNCaP and PC-3 cells. As shown in Fig. 5, in LNCaP cells the levels of survivin relative to control (1.00) was 1.09 in cells treated with docetaxel, 0.82 in cells treated with YK-4-279, and 0.48 in cells treated with the combination of docetaxel and YK-4-279. In PC-3 cells, the level of survivin relative to control (1.00) was 0.98 in cells treated with docetaxel, 0.79 in cells treated with YK-4-279, and 0.99 in cells treated with the combination.

Signal transducer and activator of transcription 3 (STAT3) is an important oncogenic protein that regulates genes involved in cell proliferation, differentiation, and invasion [32]. The constitutive activation of STAT3 has been implicated in promoting the oncogenesis and progression of prostate cancer, and it occurs frequently in primary prostate adenocarcinomas [33-34]. Some studies demonstrated that the migration and invasion of prostate cancer cells can be inhibited by suppressing the activation of STAT3 [35]. In the current study, the combination of docetaxel and YK-4-279 potently decreased the levels of p-STAT3 in both LNCaP and PC-3 cells. As shown in Fig. 5, in LNCaP cells the levels of p-STAT3 relative to control (1.00) was 0.67 in cells treated with docetaxel, 1.07 in cells treated with YK-4-279, and 0.25 in cells treated with the combination of docetaxel and YK-4-279. In PC-3 cells, the levels of p-STAT3 relative to control (1.00) were 0.70 in cells treated with docetaxel, 0.83 in cells treated with YK-4-279, and 0.31 in cells treated with the combination.

Treating $\mathrm{LNCaP}$ cells with docetaxel $(0.5 \mathrm{nM})$ or YK-4-279 $(0.5 \mu \mathrm{M})$ alone moderately decreased the levels of PSA. However, the combination of docetaxel $(0.0005 \mu \mathrm{M})$ and YK-4-279 $(0.5 \mu \mathrm{M})$ had a stronger effect on decreasing PSA levels. Specifically, PSA levels were 1.00 in control, 0.74 in cells treated with docetaxel, 0.85 in cells treated with YK-4-279, and 0.14 in cells treated with the combination of docetaxel and YK-4-279. In contrast, PSA levels were not detected in PC-3 cells with the treatments of docetaxel and YK-4-279 alone or in combination.

Treating LNCaP cells with docetaxel $(0.5 \mathrm{nM})$ or YK-4-279 $(0.5 \mu \mathrm{M})$ alone decreased the levels of AR and ETV1, and the combination treatment had no additional effect. AR levels were 1.00 in control, 0.67 in cells treated with docetaxel, 0.52 in cells treated with YK-4-279, and 0.54 in cells treated with the combination of docetaxel and YK-4-279. ETV1 levels were 1.00 in control, 0.93 in cells treated with docetaxel, 0.46 in cells treated with YK-4-279, and 0.62 in cells treated with the combination of docetaxel and YK-4-279. AR and ETV1 levels were not detected in PC-3 cells with the treatments of docetaxel and YK-4-279 alone or in combination.

\section{Discussion}

Previous studies suggested that docetaxel, the first line treatment for CRPC, has significant toxic effects and often results in resistance when used as a high-dose monotherapy for prostate cancer [36]. Based on the concept that a multitude of cellular targets may conquer drug resistance and decrease adverse effects, many studies using docetaxel-related co-treatments with one or two other drugs have been carried out; however, the effective therapies remain limited [37]. Thus, the current study aimed to identify a drug with low or no cytotoxicity that could synergistically inhibit proliferation and induce apoptosis in human prostate cancer cells when used in combination with docetaxel.

ETV1, a member of the ETS transcription factor family, can direct androgen metabolism and confer aggressive prostate cancer in targeted mice and patients [10]. ETS gene fusions play an important role of driving prostate cancer development and progression to lethal CRPC [9]. YK-4-279, a small molecule inhibitor of ETV1, can inhibit ETV1 biological activity in fusion-positive LNCaP prostate cancer cells [14-15]. In addition, YK-4-279 inhibits EWS-FLI1 activity, induces apoptosis in Ewing's sarcoma cell lines, and slows down tumor growth in mouse xenograft models [38]. YK-4-279 functions in Ewing's sarcoma cells by blocking the interaction between EWS-FLI1 and RHA. However, the mechanism by which YK-4-279 inhibit ERG- and ETV1-derived malignant phenotypes in prostate cancer cells both in vitro and in vivo is unclear [14]. In the present study, we tested the hypothesis that YK-4-279 can synergize with docetaxel to lead to greater cell death than treatment with docetaxel alone.

The current study demonstrated for the first time that the combination of docetaxel and YK-4-279 synergistically inhibits the growth of LNCaP and PC-3 prostate cancer cells. The combination of low-dose docetaxel $(0.5 \mathrm{nM})$ and YK-4-279 $(0.5 \mu \mathrm{M})$ had a more potent inhibitory effect on the growth of LNCaP and PC-3 cells than either agent used individually at a higher dose (docetaxel, $1 \mathrm{nM}$; 
YK-4-279, $1 \mu \mathrm{M}$; Fig. $1 \mathrm{C}$ and D). Moreover, the combination of low-dose docetaxel and YK-4-279 had a stronger effect on inducing apoptosis and decreasing motility and invasion in both $\mathrm{LNCaP}$ and PC-3 cells. Although PC-3 cells do not contain an ETV1 rearrangement and are androgen-independent, the combination of docetaxel and YK-4-279 still had a stronger effect on inhibiting growth, inducing apoptosis, and decreasing motility and invasion via a different mechanism in LNCaP cells than single drug alone. Overall, this study provides preclinical proof of concept that the combination of docetaxel with YK-4-279 results in a synergistic anti-tumor response in non-CRPC and CRPC models.

The AR is a transcription factor, and $A R$ activation promotes the growth and progression of prostate cancer [39]. Chromosomal translocations are frequently found in prostate cancer. For example, including ETV1 rearrangements causes the overexpression of ETV1, which cooperates with AR signaling [14]. In addition, ETV1 upregulates the expression of AR target genes as well as genes involved in steroid biosynthesis and metabolism, resulting in activation of the AR transcriptional program [10]. PSA is an androgen-regulated gene, and increased PSA levels indicate active AR signaling [40-41]. In the current study, in fusion-positive LNCaP cells YK-4-279 decreased the levels of ETV1 and AR. When used in combination with docetaxel it caused a stronger decrease in PSA levels than either drug alone. In addition, the combination of docetaxel and YK-4-279 significantly suppressed the expression of survivin, BCl-2, p-Akt, and p-STAT3 in fusion-positive LNCaP cells, suggesting that co-treatment could affect more than one signaling pathway to induce apoptosis and inhibit the growth, migration, and invasion of prostate cancer cells. In fusion-negative PC-3 cells, although co-treatment did not affect AR signaling and the levels of survivin, the levels of $\mathrm{BCl}-2$, p-Akt, p-STAT3 were significantly decreased, which explains why the combination of docetaxel and YK-4-279 could induce apoptosis and inhibit the growth, migration, and invasion of PC-3 cells.

In conclusion, the results of the current study demonstrated that the combination of low-dose docetaxel and YK-4-279 strongly inhibited growth and induced apoptosis in human prostate cancer cells. Moreover, the combination more efficiently suppressed the migration and invasion ability of PC-3 cells. These activities were accompanied by inhibition of the expression of ETV1, AR, PSA, p-STAT3, survivin, Bcl-2, and p-Akt in LNCaP cells and of p-Akt, Bcl-2, and p-STAT3 in PC-3 cells. Thus, the combination of docetaxel and YK-4-279 may be an effective approach for inhibiting the growth and metastasis of prostate cancer.

\section{Acknowledgements}

The present study was supported by funds from the Guangdong Province Leadership Grant 2011, Chinese National Science Foundation Grants ( 81272452 ), Rutgers Cancer Institute of New Jersey (CCSG P30-CA072720 RSD), and Hundred Talent Project of Guangdong University of Technology (220418008).We thank LetPub (www.letpub.com) for its linguistic assistance during the preparation of this manuscript.

\section{Competing Interests}

The authors have declared that no competing interest exists.

\section{References}

1. Roychowdhury S, Chinnaiyan AM, et al. Advancing precision medicine for prostate cancer through genomics. Journal of Clinical Oncology. 2013; 31: 1866-1873.

2. Kim TD, Jin F, Shin S, et al. Histone demethylase JMJD2A drives prostate tumorigenesis through transcription factor ETV1. The Journal of clinical investigation. 2016; 126: 706-720.

3. Long BJ, Grigoryev DN, Nnane IP, et al. Antiandrogenic effects of novel androgen synthesis inhibitors on hormone-dependent prostate cancer. Cancer research. 2000; 60: 6630-6640.

4. Cai C, Chen S, Ng P, et al. Intratumoral de novo steroid synthesis activates androgen receptor in castration-resistant prostate cancer and is upregulated by treatment with CYP17A1 inhibitors. Cancer research. 2011; 71: 6503-6513.

5. Rickman DS, Chen YB, Banerjee S, et al. ERG cooperates with androgen receptor in regulating trefoil factor 3 in prostate cancer disease progression. Neoplasia. 2010; 12: 1031-1040.

6. Nakano M, Shoji S, Higure T, et al. Low-dose docetaxel, estramustine and prednisolone combination chemotherapy for castration-resistant prostate cancer. Molecular and Clinical Oncology. 2016; 4: 942-946.

7. Sridhar SS, Freedland SJ, Gleave ME, et al. Castration-resistant prostate cancer: from new pathophysiology to new treatment. European urology. 2014; 65: 289-299.

8. Antonarakis ES, Lu C, Wang $\mathrm{H}$, et al. AR-V7 and resistance to enzalutamide and abiraterone in prostate cancer. New England Journal of Medicine. 2014; 371: 1028-1038.

9. Grasso CS, Wu YM, Robinson DR, et al. The mutational landscape of lethal, castration-resistant prostate cancer. Nature. 2012; 487: 239-243.

10. Attard G, Swennenhuis JF, Olmos D, et al. Characterization of ERG, AR and PTEN gene status in circulating tumor cells from patients with castration-resistant prostate cancer. Cancer Research. 2009; 69: 2912-2918.

11. Mehra R, Tomlins SA, Yu J, et al. Characterization of TMPRSS2-ETS gene aberrations in androgen-independent metastatic prostate cancer. Cancer research. 2008; 68: 3584-90.

12. Wu L, Zhao JC, Kim J, et al. ERG is a critical regulator of Wnt/LEF1 signaling in prostate cancer. Cancer research. 2013; 73: 6068-6079.

13. Baena E, Shao Z, Linn DE, et al. ETV1 directs androgen metabolism and confers aggressive prostate cancer in targeted mice and patients. Genes \& development. 2013; 27: 683-698.

14. Rahim S, Beauchamp EM, Kong Y, et al. YK-4-279 inhibits ERG and ETV1 mediated prostate cancer cell invasion. PloS one. 2011; 6: e19343.

15. Rahim S, Minas T, Hong SH, et al. A small molecule inhibitor of ETV1, YK-4-279, prevents prostate cancer growth and metastasis in a mouse xenograft model. PloS one. 2014; 9: e114260.

16. Mackler NJ, Pienta KJ. Drug insight: use of docetaxel in prostate and urothelial cancers. Nature clinical practice Urology. 2005; 2: 92-100.

17. Petrioli R, Francini E, Roviello G. Is there still a place for docetaxel rechallenge in prostate cancer. World journal of clinical oncology. 2015; 6: 99-103.

18. Sweeney CJ, Chen $\mathrm{YH}$, Carducci $\mathrm{M}$, et al. Chemohormonal therapy in metastatic hormone-sensitive prostate cancer. New England Journal of Medicine. 2015; 373: 737-746.

19. Gravis G, Fizazi K, Joly F, et al. Androgen-deprivation therapy alone or with docetaxel in non-castrate metastatic prostate cancer (GETUG-AFU 15): a randomised, open-label, phase 3 trial. The lancet oncology. 2013; 14: 149-158.

20. Chen X, Liu Y, Wu J, et al. Mechanistic Study of Inhibitory Effects of Atorvastatin and Docetaxel in Combination on Prostate Cancer. Cancer Genomics Proteomics 2016; 13: 151-160. 
21. Manohar SM, Padgaonkar AA, Jalota-Badhwar A, et al. Cyclin-dependent kinase inhibitor, P276-00, inhibits HIF-1a and induces G2/M arrest under hypoxia in prostate cancer cells. Prostate Cancer \& Prostatic Disease. 2012; 15: 15-27.

22. Huang $\mathrm{H}$, Cao $\mathrm{K}$, Malik $\mathrm{S}$, et al. Combination of 12-O-tetradecanoylphorbol-13-acetate with diethyldithiocarbamate markedly inhibits pancreatic cancer cell growth in 3D culture and in immunodeficient mice. International Journal of Molecular Medicine. 2015; 35: 1617-1624.

23. Nagaprashantha LD, Vatsyayan R, Singhal J, et al. Anti-cancer effects of novel flavonoid vicenin-2 as a single agent and in synergistic combination with docetaxel in prostate cancer. Biochemical pharmacology. 2011; 82: 1100-1109.

24. Liu T, Tao H, Fang Z, et al. Anti-Tumor Activity of TRPM8 Inhibitor BCTC in Prostate Cancer DU145 Cells. Oncology Letters. 2016; 11: 225-230.

25. Zheng X, Cui XX, Avila GE, et al. Atorvastatin and celecoxib inhibit prostate PC-3 tumors in immunodeficient mice. Clinical cancer research. 2007; 13: 5480-5487.

26. Huang $\mathrm{H}$, Xuan $\mathrm{C}$, Li D, et al. Combination of a-Tomatine and Curcumin Inhibits Growth and Induces Apoptosis in Human Prostate Cancer Cells. Plos One. 2015; 10: e0144293.

27. Bitting RL, Armstrong AJ. Targeting the PI3K/Akt/mTOR pathway in castration-resistant prostate cancer. Endocrine-related cancer. 2013; 20: R83-R99.

28. Quan $Y$, Wang N, Chen $Q$, et al. SIRT3 inhibits prostate cancer by destabilizing oncoprotein c-MYC through regulation of the PI3K/Akt pathway. Oncotarget. 2015; 6: 26494-26507.

29. Haldar S, Chintapalli J, Croce CM. Taxol induces bcl-2 phosphorylation and death of prostate cancer cells. Cancer Research. 1996; 56: 1253-1255.

30. Liu HY, Yu X, Liu H, et al. Co-targeting EGFR and survivin with a bivalent aptamer-dual siRNA chimera effectively suppresses prostate cancer. Scientific Reports. 2016; 6: 30346

31. Nakahara T, Kita A, Yamanaka $K$, et al YM155, a novel small-molecule survivin suppressant, induces regression of established human hormone-refractory prostate tumor xenografts. Cancer Research. 2007; 67: 8014-8021.

32. Liang $\mathrm{M}$, Zhan $\mathrm{F}$, Zhao J, et al. CPA-7 influences immune profile and elicits anti-prostate cancer effects by inhibiting activated STAT3. Bmc Cancer. 2016; 16: 504 .

33. Mora LB, Buettner R, Seigne J, et al. Constitutive activation of Stat3 in human prostate tumors and cell lines: direct inhibition of Stat 3 signaling induces apoptosis of prostate cancer cells. Cancer Research. 2002; 62: 6659-6666.

34. Canesin G, Evans-Axelsson S, Hellsten $R$, et al. The STAT3 Inhibitor Galiellalactone Effectively Reduces Tumor Growth and Metastatic Spread in an Orthotopic Xenograft Mouse Model of Prostate Cancer. European Urology. 2015; 69: 400-404.

35. Sun M, Liu C, Nadiminty $\mathrm{N}$, et al. Inhibition of Stat3 activation by sanguinarine suppresses prostate cancer cell growth and invasion. Prostate. 2012; 72:82-9.

36. Karanika S, Karantanos T, Kurosaka S, et al. GLIPR1- $\Delta$ TM synergizes with docetaxel in cell death and suppresses resistance to docetaxel in prostate cancer cells. Molecular cancer. 2015; 14: 122.

37. Attia RT, Tolba MF, Trivedi R, et al. The chemomodulatory effects of glufosfamide on docetaxel cytotoxicity in prostate cancer cells. PeerJ. 2016; 4: e2168.

38. Minas TZ, Han J, Javaheri T, et al. YK-4-279 effectively antagonizes EWS-FLI1 induced leukemia in a transgenic mouse model. Oncotarget. 2015: 6: 37678-94.

39. Darshan MS, Loftus MS, Thadani-Mulero M, et al. Taxane-induced blockade to nuclear accumulation of the androgen receptor predicts clinical responses in metastatic prostate cancer. Cancer research. 2011; 71: 6019-6029.

40. Botrel TE, Clark O, Lima Pompeo AC, et al. Efficacy and Safety of Combined Androgen Deprivation Therapy (ADT) and Docetaxel Compared with ADT Alone for Metastatic Hormone-Naive Prostate Cancer: A Systematic Review and Meta-Analysis. PloS one. 2016; 11: e0157660.

41. Chen YU, Sawyers CL, Scher HI. Targeting the androgen receptor pathway in prostate cancer. Current opinion in pharmacology. 2008; 8: 440-448. 